\title{
Optical properties of a nanoporous array in silicon
}

\author{
L. Tian, K. Bhargava Ram, I. Ahmad, L. Menon, and M. Holtz ${ }^{\text {a) }}$ \\ Department of Physics, Texas Tech University, Lubbock, Texas 79409
}

(Received 13 August 2004; accepted 22 October 2004; published online 22 December 2004)

\begin{abstract}
We demonstrate an approach for producing an array of nanopores on a silicon surface. The methods used combine nonlithographic pattern transfer and chlorine plasma etching to produce $\sim 60 \mathrm{~nm}$ diam holes up to $1 \mu \mathrm{m}$ in depth. The near-normal specular optical reflectance of these systematically modified surfaces is found to decrease dramatically with pore depth across the entire 2.0-6.0 eV photon energy range studied. We adapt an effective medium approximation to model specular reflectance taking into account diffuse scattering by the nanopatterned surface. Micro-Raman measurements show a systematic intensity increase with pore depth. The observed dependence suggests that both insertion and extraction are enhanced by the nanopatterning. (C) 2005 American Institute of Physics. [DOI: 10.1063/1.1831541]
\end{abstract}

Currently, there is substantial interest in the formation and properties of nanometer scale structures. Research in this area spans a wide array of interests in nanodots and nanowires for electronic, magnetic, and optoelectronic applications. ${ }^{1}$ In addition, photonic arrays for controlling the path of light as it travels inside waveguides are of current interest. ${ }^{2}$ Lithography has been the preferred method to patterning structures. Below $100 \mathrm{~nm}$, both deep ultraviolet (UV) and electron-beam lithography are successfully applied for patterning. The former is limited in ultimate feature size by diffraction, while the latter is limited in the total area one can practically pattern.

Recently, a nonlithographic approach to patterning has been developed. ${ }^{3}$ The approach relies on the self-assembly of a regular array of nanopores through an aluminum template under anodization. An aluminum oxide membrane is produced consisting of cylindrical arrays of nanosized pores with diameters ranging from 10 to $200 \mathrm{~nm}$. When prepared under special conditions, it has been demonstrated that the pores can exhibit a very high level of ordering. ${ }^{4}$ These two factors, small feature size and high level of ordering, make nanoporous alumina an attractive template for patterning in nanofabrication. We have recently demonstrated that this approach can be used in combination with plasma etching to transfer the nanopore pattern in the aluminum to a generic substrate. ${ }^{5}$

What has not yet been thoroughly investigated is the effect nanopores, produced on an optical surface, have on the basic optical properties of the material. Prior work has addressed extraction efficiency of organic ${ }^{6}$ and ultraviolet $(\mathrm{UV})^{7}$ light emitting diodes and reflectance properties. $^{8}$ In Ref. 8, specular reflectance was seen to drastically decrease, likely due to the irregular surface properties, which enhance diffuse reflectance. Improvements in insertion and extraction efficiencies, based on coatings or texturing, have technological implications in the areas of optoelectronic sources and

\footnotetext{
a) Author to whom correspondence should be addressed; electronic mail: mark.holtz@ttu.edu
}

photodetectors. Texturing of Si surfaces, on the micron scale, has already been applied to enhance absorbance of solar cell material. $^{9}$

In this article, we apply plasma processing to produce arrays of nanopores in silicon with smooth top surface properties. By systematically varying the etch time, different pore depths are obtained. We study the optical properties of the resulting textured surface using reflectance and Raman spectroscopies. The reflectance decreases significantly across the entire spectral range studied. We model our results using an effective medium approximation (EMA). The Raman scatter intensity exhibits a dependence that is consistent with transmission enhancement of both the excitation (insertion) and the emission (extraction) of light over the bulk starting silicon.

The nanoporous alumina template procedure has been described elsewhere. ${ }^{3}$ In this article, we use templates prepared with pores $\sim 50 \mathrm{~nm}$ in diameter. The entire template region is circular with diameter $\sim 1 \mathrm{~cm}$. Plasma etching is carried out in a commercial system employing combined inductively coupled plasma and reactive ion etching. ${ }^{5}$ The basic $\mathrm{Cl}_{2}$ etching conditions are summarized in Table I. Following the plasma etch, the alumina template is removed by a hot phosphoric and sulfuric acid wet etch. Figure 1(a) shows a plan-view scanning electron micrograph (SEM) image of the silicon surface following this procedure for a 3 min plasma process. Clearly seen is the honeycomb array of pores, which have been etched into the silicon. The pore diameter is $\sim 60 \mathrm{~nm}$, consistent with the intended value from the alumina templates. Figure 1(b) shows a SEM crosssection following etching for $2.5 \mathrm{~min}$. Vertical pore profiles are evident, verifying a highly directional etch. The aspect

TABLE I. Basic etching conditions.

\begin{tabular}{lc}
\hline \hline Plasma parameter & Value \\
\hline ICP power & $300 \mathrm{~W}$ \\
RIE power & $200 \mathrm{~W}$ \\
Chamber pressure & $8 \mathrm{mTorr}$ \\
$\mathrm{Cl}_{2} /$ Ar flow rates & $15 / 5 \mathrm{sccm}$ \\
\hline \hline
\end{tabular}




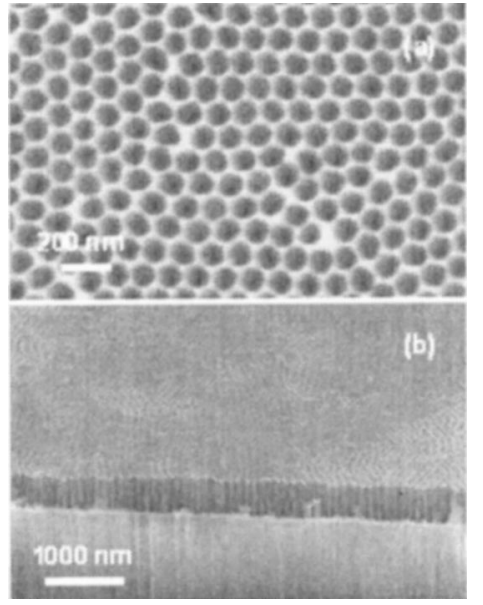

FIG. 1. SEM pictures of the nanoporous array on Si (a) top view of nanoporous array after 3 min etching. Average pore diameter $\sim 60 \mathrm{~nm}$. (b) Perspective angle view of nanoporous array after $2.5 \mathrm{~min}$ etching. Depth of pores $\sim 500 \mathrm{~nm}$.

ratio for nanopores etched into $\mathrm{Si}$ is as high as 20:1 in the experiments reported here. When considering that the etching was through the $1 \mu \mathrm{m}$ thick template plus the silicon, the process aspect ratio is significantly higher, up to 40:1 in our experiments. Cross sections such as in Fig. 1(b) were used to determine etch depth $(d)$ of the nanopores into the silicon.

Reflectance measurements were performed at a $5^{\circ}$ external angle of incidence over photon energies from 2.0 to $6.0 \mathrm{eV}$. In Fig. 2 we show measured reflectance spectra (solid curves) for the starting bulk Si wafer and after etching nanopores to several depths. The principal spectral features do not shift substantially with depth of the nanopores, indicating that the post etch Si crystal is not extensively damaged by the process. ${ }^{10}$ Interestingly, we see the reflectance to diminish across the entire range of the spectrum. We note also that the reflectance continues to decrease with depth, for the range of nanopore etch depths studied here.

Intuitively, we expect the reflectivity to decrease following etching simply because reflecting surface is removed. A straightforward linear model is, however, inadequate since the pore diameters are smaller than the wavelength of light

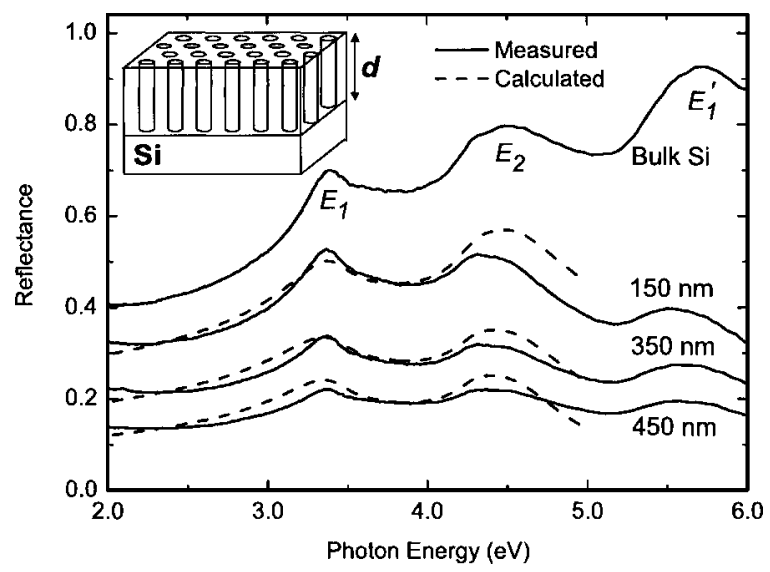

FIG. 2. Reflectance spectra of the starting bulk Si material and after etching nanopores to several depths (solid curves). Results of the EMA calculation are shown as dashed curves. (Inset) Layer construction used for the EMA model. $(\lambda)$ used for our reflectance measurements. In order to model the reflectance measurements, we adapt the MaxwellGarnett EMA. ${ }^{11}$ We note that several EMAs were examined, and exhibit similar trends to what is discussed below. According to the Maxwell-Garnett EMA, the effective complex dielectric constant $\varepsilon_{\text {eff }}$ for cylindrical shapes (pores) oriented perpendicular to a surface is given by

$$
\frac{\varepsilon_{\text {pore }}-\varepsilon_{\text {eff }}}{\varepsilon_{\text {pore }}+\varepsilon_{\text {eff }}}=f_{\text {pore }} \frac{\varepsilon_{\text {pore }}-\varepsilon_{\mathrm{Si}}}{\varepsilon_{\text {pore }}+\varepsilon_{\mathrm{Si}}},
$$

where $\varepsilon_{\mathrm{Si}}$ is the photon-energy dependent, complex dielectric function of $\mathrm{Si}$. For $\varepsilon_{\mathrm{Si}}$ we use the values from Ref. 12. Quantity $f_{\text {pore }}$ is the area fraction of pores in the nanoporous layer. The values of $f_{\text {pore }}$ are found from SEM images [Fig. 1(a)] using a straightforward contrast filter and noting that $f_{\text {pore }}$ $+f_{\mathrm{Si}}=1$. The value of $f_{\text {pore }}$ is seen to increase with process time due to a slight isotropic etch component. The dielectric function found from Eq. (1) is used to calculate the complex refractive index $\tilde{n}_{\text {eff }}$ and, subsequently, the specular reflectance $R_{\mathrm{EMA}}$ near normal incidence by considering the nanoporous layer as a thin film atop the underlying substrate, as illustrated by the inset to Fig. 2 .

Reflectance spectra calculated using this approach do not diminish as rapidly as the measured results, particularly in the UV part of the spectrum. Although the top remaining $\mathrm{Si}$ surfaces appear to be smooth after etching, there is still some roughness which will produce diffuse scattering. To account for this, we adopt a simple approach, in which incident light is reflected by a rough surface with interface width $\sigma$ which is analogous to the root mean squared surface roughness. For a transparent interface width material, calculation using the Fresnel relations results in a reflectance correction factor $-4 \pi^{2} \sigma^{2} / \lambda^{2}$ when $\sigma \ll \lambda$. In our case we must account for an opaque interface region, since the presence of this surface roughness will influence the reflectance more where optical absorption is strong. We thus weight the above correction factor with a term which represents a measure of the interaction between the light and the pore medium according to $w(d)=1-\exp (-2 \alpha d)$. This function is negligible when $\alpha$ is small (near IR and visible), and approaches unity in the UV where absorption is strong. In the following calculation, we use the optical absorption coefficient of bulk silicon, which does not differ significantly from the calculated values for the effective medium. The final specular reflectance spectrum is thus obtained using

$$
R=R_{\mathrm{EMA}}\left[1-w(d) \frac{4 \pi^{2} \sigma^{2}}{\lambda^{2}}\right],
$$

where $R$ is the reflectance calculated from the EMA. Results of this calculation are shown for different pore depths in Fig. 2 (dashed curves). Values of the surface roughness used for these calculations ranged from 18 to $32 \mathrm{~nm}$, with the larger values for deeper etches. This is reasonable, since erosion is attributed to the small plasma etch isotropy, and longer etch times lead to higher erosion. In Fig. 2 we truncate the calculated spectrum at $\sim 5 \mathrm{eV}$. The EMA calculation above this photon energy range is not valid since the wavelength approaches the pore diameter. Basic agreement between the 


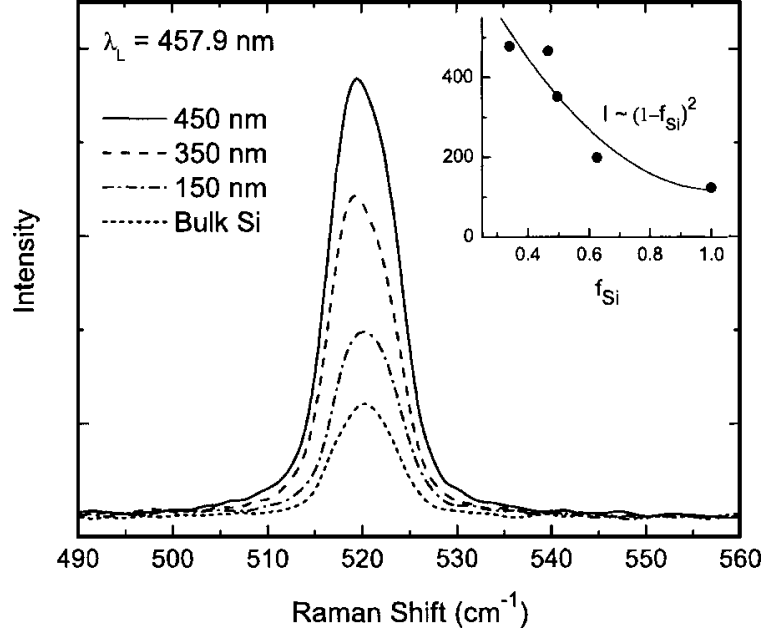

FIG. 3. Micro-Raman spectra of starting bulk Si and after etching to several pore depths. (Inset) Dependence of Raman intensity with the remaining Si area fraction.

measured and calculated spectra is excellent, suggesting that the EMA, when coupled with this approach to account for diffuse scattering, is an acceptable approach for modeling the optical properties of nanotextured surfaces.

We also examined the Raman spectra of postetched Si to determine if the decrease observed in the reflectance produces an effect on the scattering intensity. Figure 3 shows micro-Raman spectra, ${ }^{13}$ in the range of the Si $O(\Gamma)$ phonon, following etching to several pore depths. At laser excitation wavelength $457.9 \mathrm{~nm}$, the optical penetration depth $(2 \alpha)^{-1}$ is $\sim 140 \mathrm{~nm}$ in silicon. ${ }^{12}$ Thus, we probe primarily the nanoporous region. The phonon energy and line shape in Fig. 3 do not vary with etch depth, corroborating our earlier conclusion that extensive crystal damage has not occurred. Most notably, we observe a strong and reproducible intensity enhancement with pore depth. Naively, this is counterintuitive since etching reduces the volume fraction of $\mathrm{Si}$, and thus the scattering volume. This suggests an optical effect. The observed dependence is summarized in the inset to Fig. 3, where we graph intensity versus area fraction of remaining silicon. Since the reflectance drops with decreasing $f_{\mathrm{Si}}$, we expect the intensity of light entering the sample to commensurately increase. Adopting a simplified approach, the transmitted excitation intensity into the material should increase according to $T \approx(1-R) \approx\left(1-f_{\mathrm{Si}}\right)$, ignoring losses to diffuse scattering. Additionally, Raman backscatter traveling toward the surface will also encounter the nanoporous surface, suggesting $^{6}$ an additional enhancement factor for the light extraction also proportional to $(1-R) \approx\left(1-f_{\mathrm{Si}}\right)$. Combined, these two effects produce an expected intensity enhancement, over that of bulk $\mathrm{Si}$, in approximate proportion to $\left(1-f_{\mathrm{Si}}\right)^{2}$. The inset to Fig. 3 shows this dependence as the solid curve. Agreement between the observed and calculated dependence is satisfactory, confirming that the nanoporous surface enhances both insertion and extraction efficiencies.

In summary, we have systematically etched nanopores into bulk silicon with $\sim 60 \mathrm{~nm}$ pore diameter and varying depth up to $\sim 1 \mu \mathrm{m}$. Reflectance spectroscopy reveals this surface modification to provide a broadband reduction in reflectance. The reflectance is adequately described using a Maxwell-Garnett EMA which has been modified to account for roughness of the silicon surface remaining after the etch process. The model shows excellent agreement with the measurements across the valid spectral range, and reproduces the overall reduction seen in reflectance. The effects of the antireflection surface on combined insertion and extraction are studied using Raman scattering. The intensity is found to depend strongly on the remaining silicon area fraction, supporting the notion that both insertion and extraction are enhanced by the nanopore surface treatment.

\section{ACKNOWLEDGMENTS}

The authors thank J. Yun for help with the SEM images and V. Kuryatkov for assistance with the plasma etching. They also acknowledge support of this work from the National Science Foundation (Grant Nos. ECS-0323640 and ECS-0304224).

${ }^{1}$ S. Bandyopadhyay and H. S. Nalwa, Quantum Dots and Nanowires (American Scientific, Stevenson Ranch, 2003).

${ }^{2}$ A. Abidi, A. Scherer, and S. Y. Lin, Photonic Crystal Materials and Devices (SPIE, Bellingham, WA, 2003).

${ }^{3}$ L. Menon, in Quantum Dots and Nanowires, edited by S. Bandyopadhyay and H. S. Nalwa (American Scientific, Stevenson Ranch, 2003).

${ }^{4}$ H. Masuda and K. Fukuda, Science 268, 1466 (1995).

${ }^{5}$ L. Menon, K. Bhargava Ram, S. Pantibandla, D. Aurongzeb, M. Holtz, J. Yun, V. Kuryatkov, and K. Zhu, J. Electrochem. Soc. 151, C492 (2004).

${ }^{6}$ Y.-J. Lee, S.-H. Kim, J. Huh, G.-H. Kim, Y.-H. Lee, S.-H. Cho, Y.-C. Kim, and Y. R. Do, Appl. Phys. Lett. 82, 3779 (2003).

${ }^{7}$ J. Shakya, K. H. Kim, Y. J. Lin, and H. X. Jiang, Appl. Phys. Lett. 85, 142 (2004).

${ }^{8}$ Y. Kanamori, K. Hane, H. Sai, and H. Yugami, Appl. Phys. Lett. 78, 142 (2001).

${ }^{9}$ C. Wu et al., Appl. Phys. Lett. 78, 1850 (2001).

${ }^{10}$ N. Layadi, V. M. Donnelly, J. T. C. Lee, and F. P. Klemens, J. Vac. Sci. Technol. A 15, 604 (1997).

${ }^{11}$ S. Giordano, J. Electrost. 58, 59 (2002).

${ }^{12}$ D. E. Aspnes and A. A. Studna, Phys. Rev. B 27, 985 (1983).

${ }^{13}$ M. Holtz, J. C. Carty, and W. M. Duncan, Appl. Phys. Lett. 74, 2008 (1999). 